\title{
COMPARISON OF DIJKSTRA ALGORITHM WITH ANT COLONY OPTIMIZATION ALGORITHM USING RANDOM TOPOLOGY IN ALL-OPTICAL NETWORK USING RWA
}

\author{
Sachithraj T $\mathbf{T}^{\mathbf{1}}$, Piruthiviraj.P ${ }^{2}$, Preeta Sharan ${ }^{3}$ \\ ${ }^{1}$ Digital electronics and communication (M.Tech), The Oxford College of Engineering, Bangalore \\ ${ }^{2}$ Asst. Professor, Dept. of ECE, The Oxford College of Engineering, Bangalore \\ ${ }^{3}$ Associate Professor, Dept. of ECE, The Oxford College of Engineering, Bangalore
}

\begin{abstract}
All-optical networks are gaining higher and higher importance in the transmission of multimedia data. Routing and wavelength assignment is the critical issue that arises in optical networks across big infrastructures in corporate software industry. The randomness of data signals using optical networks can address specific issues to wit, blocking probability, one among the QoS parameters (which is to be reduced). Optimization techniques can be invariably applied in this scenario wherein Ant Colony Optimization (ACO) is one such method that proved very effective in solving the blocking probability issue. Routing and wavelength assignment (RWA) provides an important solution in optical networks. In this paper a comparison has been done between the results of ACO and traditional Dijkstra algorithm, in which ACO algorithm shows better performance in terms of blocking probability. Simulation results show that ACO algorithm has very low blocking probability compared with the traditional Dijkstra algorithm when the traffic load is small.
\end{abstract}

Keywords- Intelligent Optical Network; Routing and Wavelength Assignment; Ant Colony optimization Algorithm; Blocking Probability;

\section{INTRODUCTION}

Many of the technologies and techniques have emerged to enhance the use of optical fibers, aiming to its unique qualities such as very low transmission error, overall reliability, high capacity and transmission speed, and much longer distance ranges before the use of repeaters are required. Some of the multimedia applications require certain minimum requirements for good functioning and near real-time source node to destination node delivery [1].

Wavelength routing is a technology to support high-bandwidth demanding applications on the Internet. It provides all-optical data paths (routes) among the network nodes. Data is transmitted between two nodes using a lightpath, which is uniquely identified by a wavelength and a physical route (path). One of the optimization problems in optical wavelength division multiplexing networks is the routing and wavelength assignment problem [2].

The RWA problem consists of choosing the route between the two nodes in the network and assigning the wavelength for each requested lightpath in a dynamic manner where lightpaths are established on demand and released after a random holding period. This is done assuming no rerouting of existing lightpaths is allowed and with the objective of minimizing the blocking probability [3]. The performance of these algorithms directly compromises the performance of these networks [1].
This paper presents the comparison of blocking probability and simulation time for ACO algorithm with the traditional Dijkstra algorithm.

\section{ROUTING AND WAVELENGTH}

\section{ASSIGNMENT}

To carry information from source node to the destination node of an optical network, a connection must be established in the optical layer. The optical connection between a source node and a destination node is called the optical path or optical lightpath. To establish an optical path it is necessary to allocate a wavelength for each path (route). This problem is known as the routing and wavelength assignment (RWA) problem.

The blocking probability is one of the key performance parameter in the design of a RWA algorithm [4] with minimum complexity. Many studies have been reported on solution of RWA problem based on static and dynamic routing. Most of the Dynamic routing approaches perform routing and wavelength assignment separately. Generally we can split routing and wavelength assignment problem into two subproblems, one is routing problem and the other is assignment problem and then solve the two subproblems separately. 
Generally, routing algorithm consists of three major types: fixed routing (FR) algorithm, fixed-alternative routing (AFR) algorithm and adaptive routing (AR) algorithm [5].Fixed routing algorithm calculates the single data path and it is always fixed with respect to selecting the path and sending the data also. Fixed routing not only selects the path it selects the best path for transmission, so that the data transmission will take place accurately.AFR calculates for multiple data paths or channels where we can send the data through them and chooses the available path for transmission and make it as a fixed path for transmission till it get another path for transmission. AR also calculates for multiple available channels or data path while comparing and selecting the best data path depending on some expected optimal goals. The wavelength assignment algorithms are classified into four types which are most-used, least used, fixed order, and random order [6]. There are many wavelength assignment algorithms which includes Randomly Assigned algorithm (RA), First Fit algorithm (FF), Relative Capacity Loss algorithm (RCL) and Relative Lease Influence (RLI) algorithm respectively. Randomly assigned algorithm (RA) is the one in which the data paths are randomly selected. First Fit algorithm (FF) in which it will choose the data path and which ever will fit first for transmission of data will be selected. Relative Capacity Loss algorithm (RCL) and the Relative Lease Influence (RLI) algorithm will select the data path based on the specific order the wavelengths are searched. However the RA and FF are more popular because of low difficulty.

\section{ALGORITHM DESCRIPTION}

ACO is an algorithm based on the behaviour of the real ants in finding a best route (path) from a source to the food. It has been observed that the ants deposit a some amount of pheromone on its route while travelling from its nest to the food. Again while returning, the ants are subjected to follow the same path marked by the pheromone deposit and again it will deposit the pheromone in its route. In this way the ants following the optimal (shorter) route are expected to return earlier and hence increase the amount of pheromone deposit on its path at a faster rate than the ants following a longer path. ACO takes the inspiration from the foraging behaviour of the ants [7].

In all ACO algorithms, each ant gets a start node. Starting from stating node, the ant will select the next node according to algorithm rules. After visiting all nodes exactly once, the ant will return to the start node. Each ant will deposit some amount of pheromone on his route (path). The amount of pheromone depends on the quality of the ant's route: a optimal (shorter) route usually results in a greater amount of pheromone.

The ant in one node selects the next node by calculating the probabilities:

$$
p_{i j}^{k}(t)=\left\{\frac{\left[\tau_{i j}(t)\right]^{\alpha}\left[\eta_{i j}\right]^{\beta}}{\sum_{k \in \text { allowed }_{k}}\left[\tau_{i k}(t)\right]^{\alpha}\left[\eta_{i k}\right]^{\beta}}\right\}
$$

$$
j \in \text { allowed }_{k}
$$

0 otherwise Where $\eta_{i j}=1 / \mathrm{dij}$, dij is the distance between nodes $\mathrm{i}$ and node $\mathrm{j}$. In other words, the shorter the distance between two nodes $i$ and $j$ results the higher the heuristic value $\eta_{i j}$. The role of the parameters $\alpha$ and, $\beta$ is the following. If $\alpha=0$, the closest nodes are more likely to be selected. If on the contrary $\beta=0$, only pheromone amplification is at work.

The flow diagram of ACO algorithm for the Traveling Salesman Problem (TSP) is shown in Figure 1.

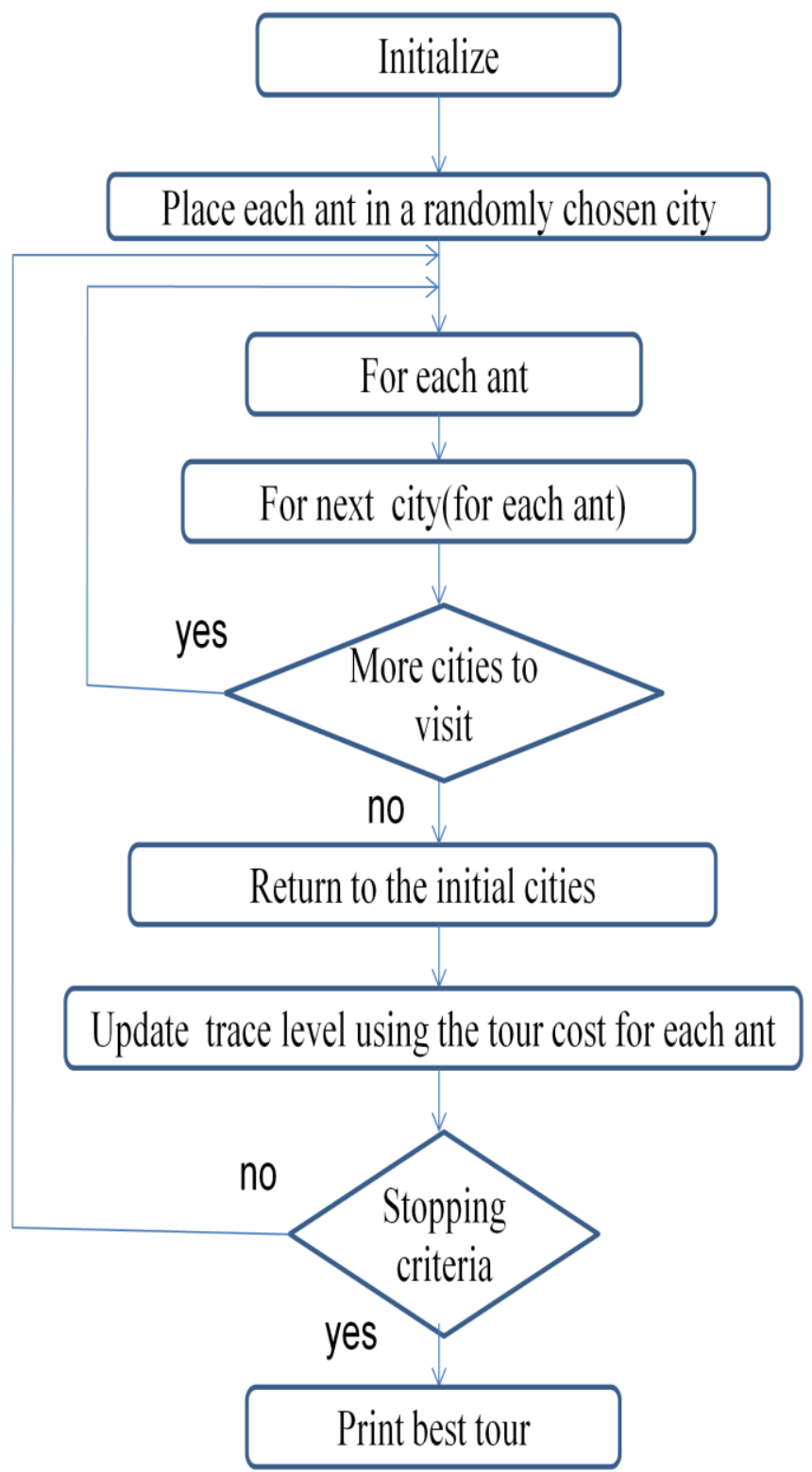

Fig 1: Flow diagram of ACO Algorithm for TSP 
Algorithm for ACO algorithm is given below:

\begin{tabular}{|l|}
\hline Loop \\
Randomly position $\mathrm{m}$ artificial ants on $\mathrm{n}$ cities \\
For city=1 to $\mathrm{n}$ \\
For ant=1 to $\mathrm{m}$ \\
\{Each ant builds a solution by adding one city after \\
the other\} \\
Select probabilistically the next city according to \\
$\quad$ exploration and exploitation mechanism \\
Apply the local trail updating rule \\
End for \\
End for \\
Apply the global trail updating rule using the best ant \\
Until End_condition
\end{tabular}

\section{SIMULATION AND ANALYSIS}

Computer simulation is used to compare the Blocking probability for ACO algorithm with traditional Dijkstra algorithm. Simulation results show that ACO algorithm has very low blocking probability compared with the traditional Dijkstra algorithm when the traffic load is small and simulation time in Random topology for ACO algorithm is very less compared with traditional dijkstra algorithm.

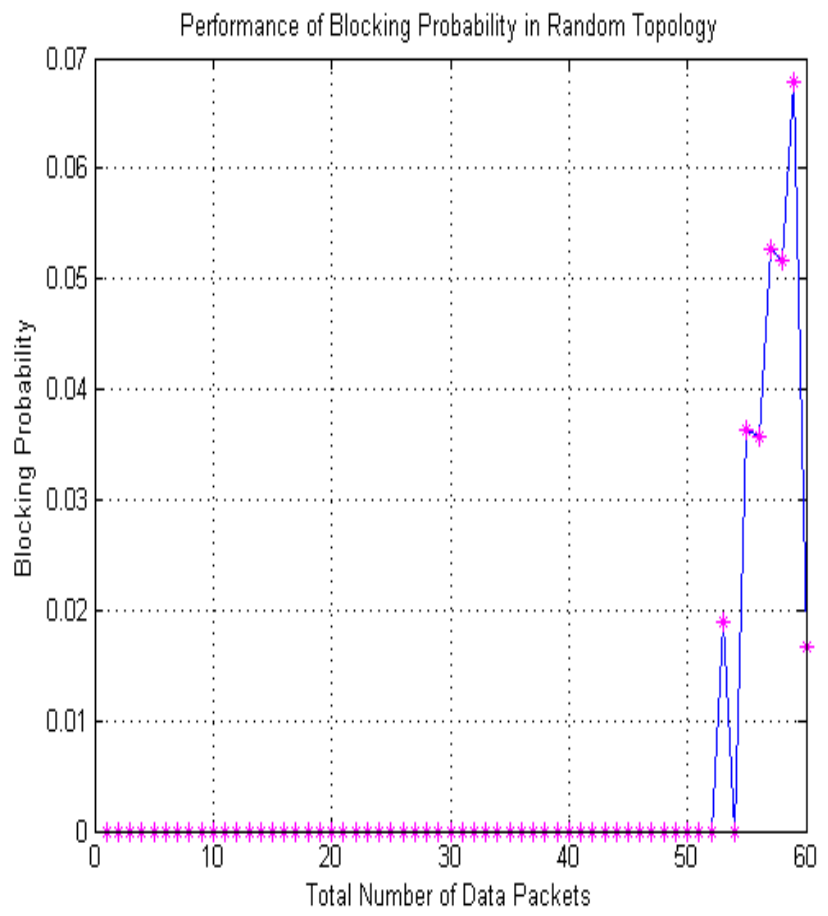

Fig 2 Blocking Probability of Dijkstra algorithm

The graph is plotted by taking Total number of data packets on $\mathrm{x}$ axis and Blocking probability on y axis. In traditional Dijkstra algorithm We have observed that the blocking probability will arises after sending 51 number of data packets and that for ACO algorithm blocking probability will arises after sending 58 number of data packets.

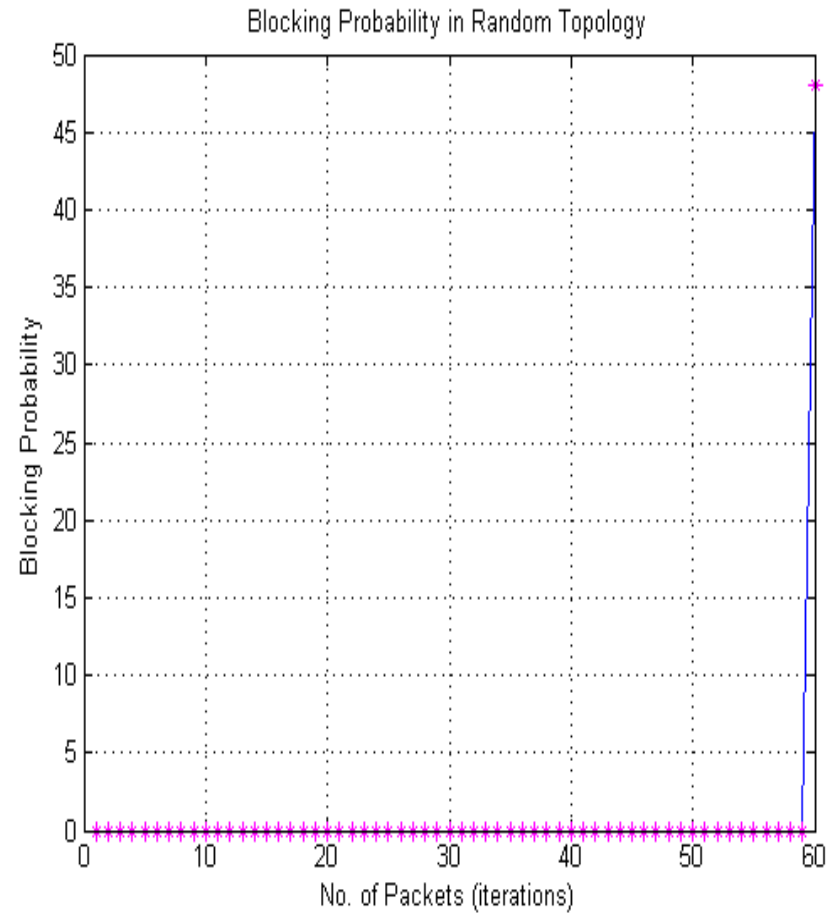

Fig 3 Blocking Probability of ACO algorithm.

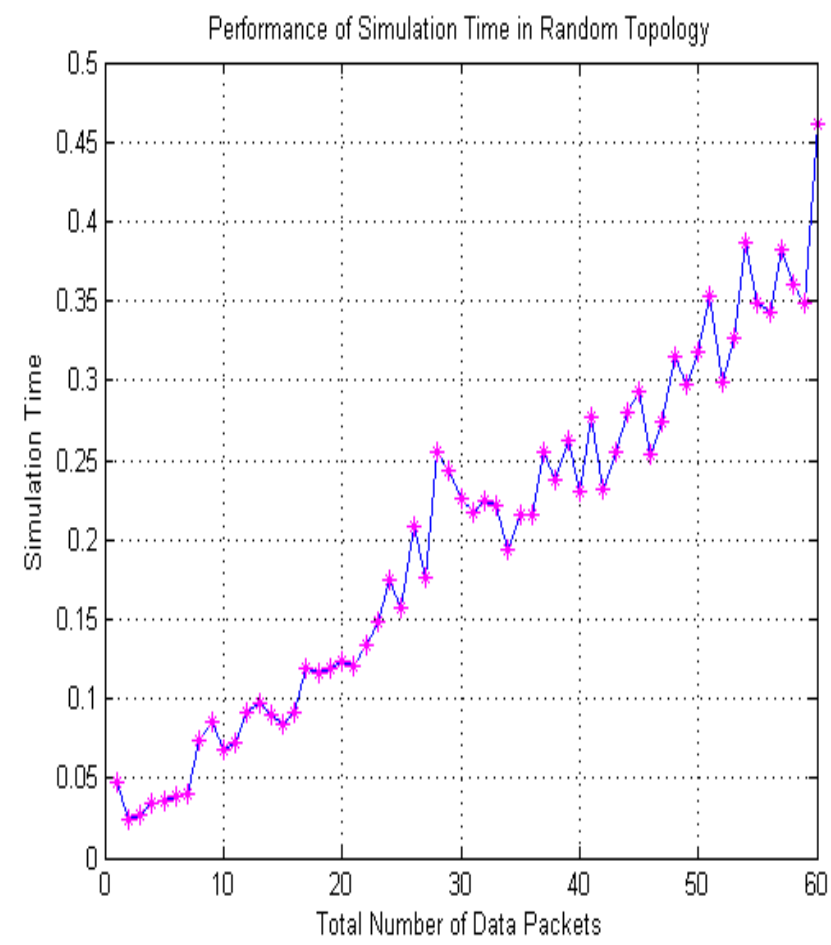

Fig 4 Simulation Time of Dijkstra algorithm 


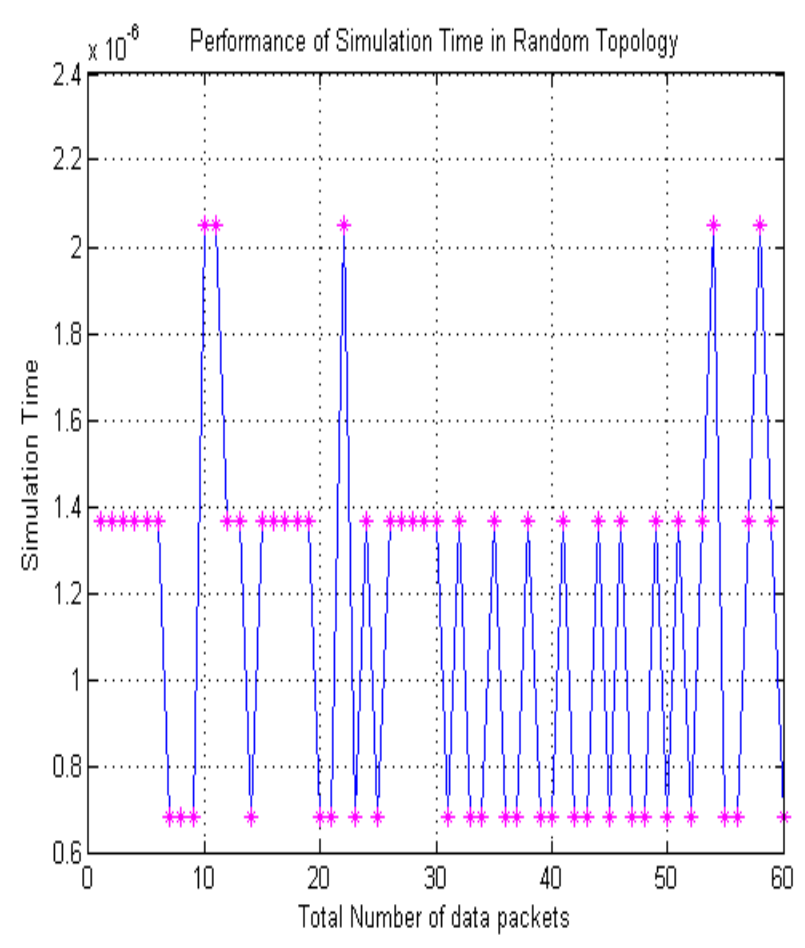

Fig 5 Simulation time of ACO algorithm

\section{CONCLUSION}

ACO algorithm is widely accepted as a promising tool to many complex optimization problems due to its good global optimization capability and parallel computing features. This paper presents an improved ACO algorithm is used to solve the RWA problem. Simulation shows that the performance of blocking probability of ACO algorithm in Random topology is very less comparing with the traditional Dijkstra algorithm and simulation time of ACO algorithm in Random topology is very less compared with traditional dijkstra algorithm simulation time will increases as the number of data packets are increased. So, we have concluded that ACO algorithm is superior to traditional Dijkstra algorithm.

\section{REFERENCES}

[1] Erick A. Donato, Joaquim Celestino Junior, Antonio S. S. Vieira, Ahmed Patel, "A Proposal of Dynamic RWA Using Ant Colony in Optical Burst Switched Networks," ICN 2012 : The Eleventh International Conference on Networks.

[2] Kavitha Bhaskaran*, Joan Triayt*, Vinod M. Vokkarane*,'Dynamic Any cast Routing and Wavelength Assignment in WDM Networks Using Ant Colony Optimization (ACO)".

[3] D.K.Kothari, S. Mahajan, S.Tiwari, "Dynamic Routing and Wavelength Assignment using Adaptive Weight Function for All - Optical WDM Network".

[4] R. A. Barry and P. A. Humblet, "Model of Blocking Probability in All-Optical Networks with and without Wavelength Changers," IEEE Journal Selected Area in Communications, 14(5):858-867, 1996.
[5] Son Hong Ngo1, Xiaohong Jiang1 and Susumu Horiguchi1,2, "An Ant-based Approach for Dynamic RWA in Optical WDM Networks,".

[6] A.Sangeetha 1 ,K.Anusudha 2 ,Shobhit Mathur 3 and Manoj Kumar Chaluvadi4 "Wavelength Assignment Problem in Optical WDM Networks," May 2009.

[7] Debasmita Mukherjee, Sriyankar Acharyya, "Ant Colony Optimization Technique Applied in Network Routing Problem" International Journal of Computer Applications (0975 - 8887) Volume 1 No. 15. 